\title{
La gestión de recursos humanos en las corporaciones locales
}

\section{Human resource management in local governments}

\author{
José Serrano Segura ${ }^{1}$ \\ MARÍA ISABEL BARBA ARAGÓN² \\ Universidad Politécnica de Cartagena (España) \\ Universidad de Murcia (España)
}

Recibido el 26 de abril de 2010, aceptado el 28 de marzo de 2011

$\mathrm{N}^{\circ}$ de clasificación JEL: M12, M50

DOI: $10.5295 /$ cdg.100233js

\section{Resumen:}

La literatura señala el importante papel que los trabajadores juegan en los resultados de una empresa y que las prácticas de gestión de recursos humanos pueden tener un efecto positivo sobre el rendimiento organizativo. Aunque esta realidad se ha estudiado ampliamente a nivel de empresa privada, lo cierto es que en el ámbito de la Administración Pública la investigación es más escasa, especialmente para corporaciones locales. Con el propósito de cubrir este hueco, este trabajo realiza un estudio de casos sobre nueve ayuntamientos de la Región de Murcia para analizar si una adecuada gestión de sus trabajadores redunda en mejoras de sus resultados a nivel organizativo, de trabajador y de prestación de servicios. La información obtenida aporta evidencia de que aquellos ayuntamientos que desarrollan prácticas de reclutamiento y selección, formación, evaluación del rendimiento y retribución teniendo en cuenta las necesidades de los puestos concretos a cubrir, el potencial de los empleados y su rendimiento, mejoran sus resultados.

Palabras clave:

Gestión de recursos humanos; Administración pública; Corporaciones locales; resultados.

\begin{abstract}
:
Literature highlights the key role that organizations' workforce has in companies' performance. It is also emphasized that human resource management is a source of competitive advantage. However, although this topic has been widely studied in the private sector, little research has focused on public administration, particularly for local governments. In order to fill this gap, this research based on a case study of nine city councils sited in the Region of Murcia, analyses if an adequate employees management is related to better organizational-level outcomes, in individuals and services. Findings suggest that the local governments that develop recruitment and

\footnotetext{
${ }_{1}^{1}$ Universidad Politécnica de Cartagena Facultad de Ciencias de la Empresa C/ Real no 330203 Cartagena (Murcia), sesejo@upct.es

${ }^{2}$ Universidad de Murcia Facultad de Economía y Empresa Campus de Espinardo 30100 Espinardo (Murcia), isabarba@um.es
} 
selection, training, performance appraisal and compensation practices taking into account the needs of the specific jobs to cover, employees' potential and their performances, improve their results.

\section{Keywords:}

Human resource management, Public administration; Local governments; Performance. 


\section{INTRODUCCIÓN}

Desde la década de los noventa el análisis de cómo las prácticas de recursos humanos influyen en los resultados de la empresa se ha convertido en un importante campo de estudio (Russell et al., 1985; Delaney y Huselid, 1996; Ichniowski et al., 1997; Ichniowski y Shaw, 1999; Ghebregiorgis y Karsten, 2007). Un creciente número de trabajos señalan que el uso de una serie de prácticas, entre las que se encuentran procesos rigurosos de reclutamiento y selección de trabajadores, formación, evaluación del rendimiento y gestión de la retribución, pueden mejorar los conocimientos y las habilidades de los empleados e incrementar su satisfacción, lo que permitirá retener a los mejores en la empresa y, en definitiva, optimizar los resultados organizativos (Huselid, 1995; Delery y Doty, 1996; Huselid et al., 1997; Zheng et al., 2006).

El interés por este tema ha aumentado gracias a los postulados de la teoría basada en los recursos. Este enfoque parte de la idea de que la empresa es un conjunto único de recursos y capacidades heterogéneas y defiende que la diferencia en la rentabilidad de las empresas se debe a sus factores internos y que son éstos, por tanto, las posibles fuentes de ventaja competitiva (Barney, 1991; Amit y Schoemacker, 1993; Youndt et al., 1996; Barney y Wright, 1998; Bae y Lawler, 2000).

Pero para que cualquiera de dichos factores pueda dar origen a una ventaja competitiva sostenible es necesario, según Barney (1991), que reúna cuatro características: debe añadir valor positivo a la empresa, debe ser único o escaso entre los competidores actuales y potenciales, debe ser imperfectamente imitable y no debe poder ser sustituido por otro recurso.

Numerosos estudios, tales como los de Wright et al. (1994), Kamoche (1996), Mueller (1996), Barney y Wright (1998) o Hitt et al. (2001), han analizado esta cuestión y han demostrado la importancia de los recursos humanos en la creación de una ventaja competitiva sostenible para la empresa.

Sin embargo, aunque la investigación del efecto que la gestión de recursos humanos tiene sobre los resultados organizativos es cada vez más amplio, la mayoría de los estudios que se han desarrollado en España se han centrado en el ámbito privado (Sanz y Sabater, 2002; Aragón et al., 2003; Jeréz et al., 2004; Ubeda, 2005; Larraza et al., 2006). Muy pocos trabajos han analizado el impacto de la gestión de recursos humanos en el campo de la Administración Pública española. Paralelamente, mientras que en las empresas privadas, desde hace ya bastante tiempo, se está realizando un importante esfuerzo por poner en marcha prácticas de recursos humanos que sirvan para optimizar el rendimiento global, en la Administración Pública sólo a partir de fechas muy recientes se empieza a hablar de "la nueva gestión pública", que persigue la creación de una administración eficiente y eficaz, favoreciendo para ello la introducción de mecanismos de competencia (García, 2007; Andreu, 2009). Ambas razones ponen de manifiesto la necesidad de realizar investigaciones en este ámbito.

Este estudio se centra, por tanto, en analizar el efecto que la gestión de recursos humanos tiene sobre los resultados de las administraciones públicas, en concreto, sobre las corporaciones locales. Para ello, en primer lugar, se realiza una revisión de la literatura. A raíz de esta revisión se plantean diversas proposiciones de investigación. A continuación se muestran los resultados de un estudio de casos realizado sobre nueve ayuntamientos de la Región de Murcia. Por último, se presentan las conclusiones, limitaciones y futuras líneas de investigación del estudio. 


\section{GESTIÓN DE RECURSOS HUMANOS Y RESULTADOS ORGANIZATIVOS}

En el estudio de las organizaciones uno de los temas más recurrentes es el de tratar de explicar las variaciones que se producen en sus resultados. De hecho, en los últimos años, la literatura ha prestado una gran atención al estudio del efecto que la gestión de recursos humanos tiene sobre los indicadores organizativos. Numerosos estudios demuestran que una eficiente gestión de las personas da lugar a mejores resultados de la empresa (Bartel, 1994; Delaney y Huselid, 1996; Delery y Doty, 1996; Ichniowski et al., 1997; Boselie et al., 2001; McElroy, 2001). Partiendo de estas investigaciones, en este trabajo se han identificado cuatro prácticas de recursos humanos que generalmente tienen un impacto significativo sobre los resultados organizativos: reclutamiento y selección, formación, evaluación del rendimiento y retribución. Se espera que dichas prácticas influyan de forma positiva, no sólo en las empresas privadas sino que también lo hagan en la Administración Pública.

\subsection{Reclutamiento y selección}

El reclutamiento es, según Gómez-Mejía et al. (2001), el primer paso del proceso de contratación y supone la reserva de candidatos para un determinado puesto de trabajo. Por su parte, definen la selección como el proceso mediante el cual se decide si se va a contratar, o no, a cada uno de los candidatos al puesto. Según Pfeffer (1995), un proceso riguroso de reclutamiento y selección genera altas expectativas de un buen rendimiento y manda un mensaje de la importancia que la organización concede a sus empleados.

Los estudios realizados sobre muestras de empresas privadas ponen de manifiesto que un uso eficiente de estas prácticas influye favorablemente en los resultados organizativos (Delaney y Huselid, 1996), incidiendo en variables tales como los beneficios (Terpstra y Rozell, 1993) o la productividad (Huselid, 1995; Koch y McGrath, 1996; Ichniowski et al., 1997; Ichniowski y Shaw, 1999).

En el caso de la Administración Pública española existen tres sistemas posibles de selección: la oposición, el concurso-oposición y el concurso. Sin embargo, en ninguno se sigue el enfoque de la gestión por competencias lo que ha dado lugar a que, en muchas ocasiones, las personas elegidas hayan acreditado la mejor puntuación en los conocimiento y experiencia requeridos pero no en las actitudes y valores necesarios para desempeñar su puesto con éxito (Parrés, 2005).

Si el reclutamiento y la selección son procesos claves para aprovisionarse de las cualificaciones y competencias necesarias, en el caso de puestos de trabajo público lo son mucho más porque la permanencia de los seleccionados va a ser, prácticamente, indefinida. En consecuencia, estos procesos deberían mejorarse radicalmente. Así, según Udechukwu y Manyak (2009), el primer paso que debería dar cualquier organización pública en un proceso de contratación sería la realización de un análisis del puesto de trabajo a cubrir. Otro paso preliminar debería ser comunicar adecuadamente la información sobre las tareas a desempeñar. Dicha información debería ser específica y centrarse en los conocimientos, habilidades y responsabilidades inherentes al puesto con la intención de atraer a un alto porcentaje de candidatos cualificados (Mastracci, 2009). Por su parte, Villoria y del Pino (2009) indican que para que la oferta de empleo público en España pueda cubrir sus obje- 
tivos debería incluir programas de reclutamiento activo destinados a captar candidatos que compartan los valores de lo público y cuya actitud hacia el trabajo y su responsabilidad hacia los problemas colectivos sean los adecuados.

Por ello, aquellas administraciones que a la hora de reclutar y seleccionar, aparte de cumplir la legalidad vigente, se preocupan por buscar una buena adecuación entre el puesto y la persona que lo va a desempeñar suelen obtener una mayor y más rápida integración de los empleados en la organización, mayor motivación y satisfacción del trabajador y mejora de la imagen de la administración ante el ciudadano (Sáenz Blanco, 2004). Teniendo en cuenta esto se plantea la primera proposición de investigación:

Proposición 1: las administraciones públicas que en sus procesos de reclutamiento y selección tienen en cuenta las competencias concretas de los puestos a cubrir obtienen mejores resultados que las que no lo hacen.

\subsection{Formación}

La formación se define como un conjunto de actividades cuyo propósito es mejorar el rendimiento presente y futuro del empleado, aumentando su capacidad a través de la mejora de sus conocimientos, habilidades y actitudes (Dolan et al., 2003).

Tradicionalmente se ha considerado que la formación de los trabajadores tiene un efecto positivo sobre los resultados organizativos. Esta suposición se basa en el hecho de que la formación juega un papel clave en el desarrollo de dos de las principales fuentes de ventaja competitiva de la empresa: su capital humano (Bartel, 1994; Raghuram, 1994) y su conocimiento organizativo (Alavi y Leidner, 2001; Bollinger y Smith, 2001).

Sin embargo, no ha sido hasta aproximadamente los años 90 cuando se han empezado a realizar investigaciones para analizar dicho efecto y, aunque algunos estudios empíricos no han encontrado resultados significativos (Black y Lynch, 1996; Schonewille, 2001), en general se ha demostrado que la formación tiene un efecto positivo sobre la productividad (Barron y Berger, 1999; Faems et al., 2005; Birdi et al., 2008), sobre las ventas (Huselid, 1995; Barrett y O'Connell, 2001), sobre los sueldos de los trabajadores (Bartel, 1994; Lengermann, 1996) y sobre la calidad (Holzer et al., 1993; Kidder y Rouiller, 1997; Murray y Raffaele, 1997).

En el caso de la Administración Pública, la formación debe tener como objetivo adaptarse a las necesidades cambiantes de los ciudadanos a los que se prestan los servicios, manteniendo actualizados los conocimientos y las habilidades del personal (Gonzalo y Cortés, 2004). De acuerdo con el estudio de casos realizado por Coggburn y Hays (2003), aquellos ayuntamientos que muestran un fuerte compromiso con la formación consiguen mejorar la satisfacción en el trabajo y aumentar la calidad de vida de la mayoría de sus empleados. De hecho, aquellos municipios en los que el programa de formación se diseña partiendo de un correcto análisis de necesidades y teniendo en cuenta las habilidades esenciales que deben desempeñarse en cada puesto de trabajo son los que mejores resultados alcanzan (Gabris et al., 1998).

Según la experiencia llevada a cabo en el departamento de formación de la Diputación Provincial de Alicante, aspectos tales como un adecuado análisis de las necesidades formativas, buena calidad metodológica y seguimiento de los resultados logrados a nivel de aprendizaje y de transferencia, consiguen mejorar la satisfacción de los empleados y 
su rendimiento en el trabajo (Latorre, 2003). A la vista de estos argumentos se plantea la segunda proposición de investigación:

Proposición 2: las administraciones públicas que en sus planes de formación tienen en cuenta las necesidades concretas del puesto de trabajo obtienen mejores resultados que las que no lo hacen.

\subsection{Evaluación del rendimiento}

La evaluación del rendimiento consiste en la identificación, medición y gestión del desempeño humano en la empresa (Gómez-Mejía et al., 2001). Esta práctica trata de descubrir en qué medida es productivo un empleado y si podrá mejorar su rendimiento futuro (Dolan et al., 2003).

Cuando una empresa lleva a cabo una evaluación del rendimiento de sus trabajadores persigue objetivos tanto administrativos como motivacionales, buscando, en última instancia, una mejora de sus resultados organizativos. La literatura que ha analizado el efecto de la evaluación del desempeño demuestra que si esta práctica reúne las características necesarias es posible conseguir dicha mejora (Martin y Bartol, 1998; Mani, 2002; Khoury y Analoui, 2004; Caruth y Humphreys, 2008). El problema surge cuando la empresa la convierte simplemente en una medida de control. En este caso sus efectos son contraproducentes y algunos estudios abogan por su eliminación (Bowman, 1994; Gray, 2002).

En la Administración Pública la evaluación del rendimiento a nivel individual es muy novedosa (Sole y Schiuma, 2010) ya que tradicionalmente se ha empleado el concepto de "responsabilidad administrativa", basado en el cumplimiento formal de las normas. Sin embargo, hoy en día se impone la necesidad de responder por la utilización eficiente de los recursos y por el grado de cumplimiento de los objetivos y se están empezando a establecer medidas para conocer el proceso de prestación de servicios públicos, contrastarlo con estándares de economía, desempeño, equidad, eficacia y eficiencia e imputar a los gestores los resultados conseguidos (Gonzalo y Cortés, 2004). Siguiendo a Villoria y del Pino (2009), se puede decir que existe una preocupación general por mejorar el rendimiento del sector público y, en consecuencia, por medirlo. De hecho, la presión para evaluar el rendimiento de los empleados públicos es si cabe mayor a nivel local dada la cercanía entre trabajadores y políticos por lo que se hace necesario diseñar mejores medidas del rendimiento más relacionadas con el puesto a desempeñar (Gabris et al., 1998).

Según Coggburn y Hays (2003), muchas iniciativas en la administración fracasan por la falta de objetivos claros y por asignaciones ambiguas. Por ello, para que los planes de evaluación del rendimiento alcancen un éxito global deben establecer claramente las metas a alcanzar y fijar las responsabilidades a nivel individual (Ufuk, 2007).

En esta línea, en España, el nuevo Estatuto del Empleado Público indica que las Administraciones Públicas establecerán sistemas que permitan la evaluación del desempeño de sus empleados, adecuando los sistemas de valoración a criterios de transparencia, objetividad, imparcialidad y no discriminación, aplicándose sin menoscabo de los derechos de los empleados públicos.

Diversos autores han analizado el valor de los sistemas de evaluación del rendimiento, las barreras a su implantación y los resultados derivados de su utilización en la administra- 
ción pública (Ammons, 1995; Kravchuch y Schack, 1996, De Lancer Julnes y Holzer, 2003; Sanger, 2008). Entre otras conclusiones obtienen que esta práctica mejora la satisfacción y motivación de los empleados, lo que repercute a su vez en una mejora del servicio ofrecido a la ciudadanía y en un abaratamiento de los costes (Sáenz Blanco, 2004). Asimismo, introduce nuevas actitudes hacia el servicio público, mejora la comunicación vertical, clarifica papeles y responsabilidades, aumentando, en definitiva, la eficiencia (Villoria y del Pino, 2009). Teniendo en cuenta estos argumentos se plantea la tercera proposición de investigación:

Proposición 3: las administraciones públicas que responsabilizan a los empleados públicos de la realización de su trabajo y evalúan su potencial obtienen mejores resultados que las que no lo hacen.

\subsection{Gestión de la retribución}

En sentido amplio, la retribución total se refiere al conjunto de recompensas intrínsecas y extrínsecas que el empleado recibe por desempeñar su trabajó (Martocchio, 1998). Las recompensas intrínsecas o no monetarias consisten en la satisfacción que una persona recibe del puesto mismo o del entorno del puesto en el que trabaja y engloba aspectos tales como reconocimiento y estatus, buena comunicación, ascensos, mayor autonomía, etc. Las recompensas extrínsecas o monetarias consisten en el pago que recibe una persona en forma de salarios, sueldos, incentivos y prestaciones a cambio de su trabajo (Mondy et al., 1999; Henderson, 2003). Toda retribución debe atraer potenciales candidatos, retener a los buenos empleados y servir de elemento motivador, pero además ha de ser flexible y adaptable al valor que tiene cada individuo para la organización (Lawler, 1990; De la Calle y Ortiz, 2004).

Numerosos estudios han analizado el impacto de las retribuciones en los resultados de la empresa y han llegado a la conclusión de que una gestión avanzada de las compensaciones puede ser una fuente de ventaja competitiva sostenible (Ichniowski et al., 1997; Ichniowski y Shaw, 1999; Chiu et al., 2002). Además, los incentivos salariales mejoran los resultados organizativos, reducen la rotación de los trabajadores e incrementan las ventas (Arthur, 1994; Delaney y Huselid, 1996; Batt, 2002).

En el caso de la Administración Pública la retribución suele fallar en el objetivo de ser un elemento motivador. Actualmente, se impone revisar el actual sistema para dar cabida a una compensación que posibilite la evaluación del desempeño (Gonzalo y Cortés, 2004). Para el buen funcionamiento de la Administración son necesarios el compromiso y la implicación de sus empleados y esto depende, en gran medida, de la retribución (Parrés, 2005). Según Luna (2004), el futuro debe venir marcado por una individualización de las retribuciones basadas en una evaluación del trabajo desarrollado y por una necesaria equidad externa. En esta línea de la equidad, algunos estudios realizados en Estados Unidos analizan si una retribución comparable o superior a la del sector privado aumenta la probabilidad de que determinados profesionales (tales como programadores informáticos o ingenieros) opten por trabajar en el gobierno federal y de que se reduzca su tasa de abandono, obteniendo la mayoría relaciones positivas aunque débiles entre salarios y permanencia en la administración (Lewis, 1991; Kim, 2005; Mastracci, 2009).

Según Rusaw (2009), para que la gestión de las retribuciones basada en el rendimiento mejore la motivación, las competencias y los resultados, no se debe olvidar ponerla en 
relación con competencias profesionales amplias que permitan a un tiempo mejorar los resultados de la administración y mejorar la eficiencia individual. Tomando como referencia estos argumentos se plantea la cuarta proposición de investigación:

Proposición 4: las administraciones públicas que gestionan la retribución de sus empleados relacionándola con la evaluación del rendimiento obtienen mejores resultados que las que no lo hacen.

\section{ESTUDIO DE CASOS. METODOLOGÍA}

Según algunos autores como Darke et al. (1998), la metodología del estudio de casos es una buena forma de investigar fenómenos poco conocidos, muy complejos y dinámicos y es la más adecuada cuando las investigaciones empíricas sobre un tema aún son escasas. Teniendo en cuenta las peculiaridades que presenta la gestión de recursos humanos en la Administración Pública con respecto al sector privado y los pocos estudios que se han realizado sobre el tema en España, se ha considerado que es la metodología ideal para abordar este trabajo en la actualidad.

\subsection{Muestra y recogida de información}

La población objeto del presente estudio está compuesta por los 45 ayuntamientos de la Región de Murcia. Estos se han clasificado en función del número de habitantes obteniendo: 9 ayuntamientos de menos de 5.000 habitantes, 10 de entre 5.000 y $10.000,13$ de entre 10.000 y $20.000,9$ entre 20.000 y 50.000 y 4 de más de 50.000 habitantes. Se ha procedido a esta agrupación por entender que existe una relación entre el número de habitantes del municipio y las necesidades de recursos humanos en sus ayuntamientos.

En total se han realizado nueve estudios de casos. Para su elección se ha tenido en cuenta el número de municipios que se encuadran en cada grupo de población. Se han escogido dos ayuntamientos en cada grupo menos en el último (poblaciones de más de 50.000 habitantes) donde sólo se ha realizado un estudio debido al menor número de ayuntamientos que lo componen. En el estudio de casos no es necesario una elección aleatoria de la muestra (Yin, 1994), por ello los casos se han escogido en función de las posibilidades de colaboración de las distintas corporaciones locales.

En concreto los nueve estudios de casos se han realizado sobre los siguientes ayuntamientos de la Región de Murcia: ayuntamientos de menos de 5.000 habitantes (caso 1: Ayuntamiento de Villanueva del Segura; caso 2: Ayuntamiento de Albudeite), ayuntamientos de entre 5.000 y 10.000 habitantes (caso 3: Ayuntamiento de Lorquí; caso 4: Ayuntamiento de Abanilla), ayuntamientos de entre 10.000 y 20.000 habitantes (caso 5: Ayuntamiento de Alhama de Murcia; caso 6: Ayuntamiento de Torres de Cotillas), ayuntamientos de entre 20.000 y 50.000 habitantes (caso 7: Ayuntamiento de San Javier; caso 8: Ayuntamiento de Cieza), ayuntamientos de más de 50.000 habitantes (caso 9: Ayuntamiento de Cartagena - caso piloto).

La recogida de información se ha realizado entre enero y febrero de 2006. Para ello, siempre que ha sido posible se han realizado entrevistas personales con el responsable de 
recursos humanos del ayuntamiento. Cuando alguna razón ha imposibilitado el encuentro personal se ha empleado el correo electrónico y el correo postal. En todos los casos para guiar la entrevista se ha utilizado un cuestionario testado previamente por varios técnicos del servicio de recursos humanos del ayuntamiento de Cartagena, ya que este ayuntamiento fue el utilizado como caso piloto.

\subsection{Medición de las variables y análisis de la información}

En relación con las variables independientes, para conocer en qué medida se han llevado a cabo las diferentes prácticas de recursos humanos en cada uno de los ayuntamientos estudiados, se ha pedido a los encuestados que indiquen su grado de acuerdo con las siguientes afirmaciones, medidas en una escala de 5 puntos, donde 1 representa total desacuerdo con la afirmación y 5 total acuerdo:

- Al programar los procesos de reclutamiento y selección se tienen en cuenta las competencias concretas de los puestos a cubrir.

- Al diseñar los planes de formación se tienen en cuenta las necesidades concretas del puesto de trabajo.

- Se responsabiliza a los empleados públicos de la realización de su trabajo.

- Se evalúa el potencial de los empleados públicos para motivarlos y mejorar su rendimiento.

- La retribución de los empleados públicos se relaciona con la evaluación de su rendimiento.

En relación con las variables dependientes, para recoger información de los resultados del ayuntamiento se ha valorado su actuación en tres escenarios distintos. Se sigue así la forma de proceder de algunas experiencias previas en gestión de recursos humanos a nivel de corporación local como es el caso de la del ayuntamiento de Manlleu (Sáenz Blanco, 2004). Para conocer en qué grado se han producido los resultados se ha pedido a los encuestados que indiquen, utilizando una escala de 5 puntos, donde 1 representa fuerte empeoramiento y 5 fuerte mejora, cómo han evolucionado los siguientes indicadores en su ayuntamiento en los dos últimos años:

- Resultados a nivel de organización: gasto público; número de accidentes de trabajo; relaciones con los compañeros, cultura corporativa; clima laboral.

- Resultados a nivel de trabajador: absentismo; motivación; identidad y compromiso con el ayuntamiento; capacidad de los empleados para solucionar problemas y afrontar cambios.

- Resultados a nivel de prestación de servicios: calidad del servicio ofertado; satisfacción de los ciudadanos; tiempo de respuesta al ciudadano.

A la hora de trabajar con los resultados de cada ayuntamiento se ha calculado el valor medio de las puntuaciones otorgadas a cada uno de los indicadores que forman los distintos escenarios.

Por último, señalar que para realizar los análisis de la evidencia que los casos proporcionan a las proposiciones planteadas, se han tabulado los datos, utilizando la técnica del pattern matching, y se ha estudiado la información cualitativa recogida de las entrevistas. A continuación se presentan los principales resultados obtenidos referentes a cómo la gestión de los recursos humanos influye en los resultados de los ayuntamientos. 


\section{ESTUDIO DE CASOS. RESULTADOS}

En la tabla 1 se recogen algunos datos generales de los ayuntamientos que corresponden a los nueve estudios de casos y que pueden ayudar a interpretar algunos de los resultados que se alcanzan en esta investigación.

Tabla 1

Datos generales de los ayuntamientos

\begin{tabular}{lccccccccc}
\hline \multicolumn{1}{c}{ CASO } & 1 & 2 & 3 & 4 & 5 & 6 & 7 & 8 & 9 \\
\hline Antigüedad & $>30$ & $>30$ & $>30$ & $>30$ & $>30$ & $>30$ & $>30$ & $>30$ & $>30$ \\
$\mathrm{~N}^{\circ}$ Funcionarios & 10 & 4 & 42 & 18 & 107 & 87 & 154 & 114 & 1035 \\
$\mathrm{~N}^{\circ}$ Laborales & 7 & 6 & 11 & 42 & 105 & 124 & 280 & 150 & 150 \\
Edad Funcionarios & 40 & 35 & 41 & 47 & 40 & 45 & 43 & 36 & 40 \\
Edad Laborales & 30 & 40 & 33 & 55 & 35 & 39 & 41 & 38 & 35 \\
Población Derecho & 1.800 & 1.400 & 6.498 & 6.265 & 19.669 & 19.000 & 28.664 & 34.000 & 203.945 \\
Población Flotante & 1.900 & 2.000 & --- & --- & --- & 23.000 & 43.000 & 38.000 & 353.945 \\
\hline
\end{tabular}

Fuente: Elaboración propia.

A continuación, se procede a comprobar si la información extraída del estudio de casos aporta o no evidencia empírica a las proposiciones planteadas. En la tabla 2 se muestran los datos obtenidos en cada uno de los ayuntamientos respecto a las prácticas de recursos humanos y a las categorías de resultados.

Tabla 2

Efecto de las prácticas de recursos humanos sobre los resultados

\begin{tabular}{ccccccccc}
\hline CASO & $\begin{array}{c}\text { Reclutamiento } \\
\text { y selección }\end{array}$ & Formación & $\begin{array}{c}\text { Responsabilidad } \\
\text { empleados }\end{array}$ & $\begin{array}{c}\text { Evaluación } \\
\text { potencial }\end{array}$ & Retribución & $\begin{array}{c}\text { Resultados } \\
\text { organización }\end{array}$ & $\begin{array}{c}\text { Resultados } \\
\text { trabajador }\end{array}$ & $\begin{array}{c}\text { Resultados } \\
\text { prestación } \\
\text { servicios }\end{array}$ \\
\hline 1 & 5 & 5 & 3 & 3 & 3 & 4,6 & 5,0 & 5,0 \\
2 & 3 & 3 & 3 & 3 & 2 & 3,2 & 3,0 & 3,0 \\
3 & 4 & 4 & 3 & 3 & 2 & 3,0 & 3,25 & 3,0 \\
4 & 2 & 2 & 3 & 2 & 3 & 2,0 & 2,0 & 2,0 \\
5 & 5 & 4 & 3 & 1 & 1 & 2,6 & 3,0 & 3,33 \\
6 & 5 & 4 & 4 & 4 & 4 & 3,0 & 3,0 & 4,0 \\
7 & 1 & 2 & 1 & 1 & 2 & 1,2 & 2,25 & 2,6 \\
8 & 5 & 4 & 4 & 4 & 3 & 3,6 & 3,75 & 3,66 \\
9 & 2 & 4 & 1 & 1 & 1 & 1,4 & 2,25 & 1,0 \\
\hline
\end{tabular}

Fuente: elaboración propia. 
La primera proposición de investigación establece que las administraciones públicas que en sus procesos de reclutamiento y selección tienen en cuenta las competencias concretas de los puestos a cubrir obtienen mejores resultados que las que no lo hacen. En la tabla 3 se comenta cada caso de estudio, indicando si se obtiene evidencia o no sobre la proposición planteada.

Tabla 3

\section{Evidencia sobre la proposición de investigación 1}

\begin{tabular}{|c|c|}
\hline CASO & Evidencia \\
\hline 1 & $\begin{array}{l}\text { Se obtiene evidencia. Los procesos de reclutamiento y selección que realiza este ayun- } \\
\text { tamiento siempre tienen en cuenta las competencias concretas de los puestos a cubrir y } \\
\text { se observa una gran mejoría de los resultados. }\end{array}$ \\
\hline 2 & No se puede afirmar la existencia de evidencia en uno u otro sentido. \\
\hline 3 & No se puede afirmar la existencia de evidencia en uno u otro sentido. \\
\hline 4 & $\begin{array}{l}\text { Se obtiene evidencia. Los procesos de reclutamiento y selección que realiza este } \\
\text { ayuntamiento raras veces tienen en cuenta las competencias concretas de los puestos a } \\
\text { cubrir y se observa un empeoramiento de los resultados en los tres campos analizados. }\end{array}$ \\
\hline 5 & $\begin{array}{l}\text { No se obtiene evidencia. Los procesos de reclutamiento y selección que realiza este } \\
\text { ayuntamiento siempre tienen en cuenta las competencias concretas de los puestos a } \\
\text { cubrir y sólo se observa una ligera mejoría de los resultados a nivel de prestación de } \\
\text { servicios mientras que los resultados a nivel de organización empeoran. }\end{array}$ \\
\hline 6 & $\begin{array}{l}\text { Se obtiene evidencia. Los procesos de reclutamiento y selección que realiza este ayun- } \\
\text { tamiento siempre tienen en cuenta las competencias concretas de los puestos a cubrir y } \\
\text { se observa una mejoría de los resultados a nivel de prestación de servicios. }\end{array}$ \\
\hline 7 & $\begin{array}{l}\text { Se obtiene evidencia. Los procesos de reclutamiento y selección que realiza este } \\
\text { ayuntamiento casi nunca tienen en cuenta las competencias concretas de los puestos } \\
\text { a cubrir y se observa un gran empeoramiento de los resultados sobre todo a nivel de } \\
\text { organización. }\end{array}$ \\
\hline 8 & $\begin{array}{l}\text { Se obtiene evidencia. Los procesos de reclutamiento y selección que realiza este ayun- } \\
\text { tamiento siempre tienen en cuenta las competencias concretas de los puestos a cubrir y } \\
\text { se observa una gran mejoría de los resultados. }\end{array}$ \\
\hline 9 & $\begin{array}{l}\text { Se obtiene evidencia. Los procesos de reclutamiento y selección que realiza este ayun- } \\
\text { tamiento casi nunca tienen en cuenta las competencias concretas de los puestos a cubrir } \\
\text { y se observa un gran empeoramiento de los resultados en los tres campos analizados. }\end{array}$ \\
\hline
\end{tabular}

Fuente: elaboración propia.

Con respecto a la primera proposición, los participante en el estudio de casos, consideran que es muy importante que a la hora de contratar a nuevos empleados se les ofrezca una información real sobre el tipo de organización y el puesto de trabajo con que se van a encontrar para que no se produzca un choque entre sus expectativas y la realidad que pueda desmotivarlos. Se hace también necesario, desde el punto de vista de los casos analizados, 
cambiar el sistema tradicional de puestos de trabajo ya que este sistema confiere una gran rigidez a la Administración al limitar a los empleados y dificultar la asignación de nuevas tareas, funciones y roles. En su opinión, la clave del éxito se encuentra quizás en una reducción radical del número de puestos para garantizar así la flexibilidad, convirtiendo un gran número de puestos especializados en un menor número de puestos genéricos. Igualmente, defienden que para mantener el estatus funcionarial como garantía de seguridad en el empleo, objetividad e independencia, las condiciones laborales deben flexibilizarse y adaptarse a las necesidades municipales en función de las nuevas exigencias del entorno económico y social.

La segunda proposición de investigación establece que las administraciones públicas que en sus planes de formación tienen en cuenta las necesidades concretas del puesto de trabajo obtienen mejores resultados que las que no lo hacen. En la tabla 4 se comenta cada caso de estudio, indicando si se obtiene evidencia o no sobre la proposición planteada.

Tabla 4

\section{Evidencia sobre la proposición de investigación 2}

\begin{tabular}{|c|c|}
\hline CASO & Evidencia \\
\hline 1 & $\begin{array}{l}\text { Se obtiene evidencia. Los planes de formación que realiza este ayuntamiento siempre } \\
\text { tienen en cuenta las necesidades concretas del puesto de trabajo y se observa una gran } \\
\text { mejoría de los resultados. }\end{array}$ \\
\hline 2 & No se puede afirmar la existencia de evidencia en uno u otro sentido. \\
\hline 3 & No se puede afirmar la existencia de evidencia en uno u otro sentido. \\
\hline 4 & $\begin{array}{l}\text { Se obtiene evidencia. Los planes de formación que realiza este ayuntamiento raras } \\
\text { veces tienen en cuenta las necesidades concretas del puesto de trabajo y se observa un } \\
\text { empeoramiento de los resultados. }\end{array}$ \\
\hline 5 & $\begin{array}{l}\text { No se obtiene evidencia. Los planes de formación que realiza este ayuntamiento nor- } \\
\text { malmente tienen en cuenta las necesidades concretas del puesto de trabajo y se observa } \\
\text { una ligera mejoría de los resultados a nivel de prestación de servicios y un empeora- } \\
\text { miento de los resultados a nivel de organización. }\end{array}$ \\
\hline 6 & $\begin{array}{l}\text { Se obtiene evidencia. Los planes de formación que realiza este ayuntamiento normal- } \\
\text { mente tienen en cuenta las necesidades concretas del puesto de trabajo y se observa una } \\
\text { mejoría de los resultados a nivel de prestación de servicios. }\end{array}$ \\
\hline 7 & $\begin{array}{l}\text { Se obtiene evidencia. Los planes de formación que realiza este ayuntamiento raras } \\
\text { veces tienen en cuenta las necesidades concretas del puesto de trabajo y se observa un } \\
\text { gran empeoramiento de los resultados sobre todo a nivel de organización. }\end{array}$ \\
\hline 8 & $\begin{array}{l}\text { Se obtiene evidencia. Los planes de formación que realiza este ayuntamiento normal- } \\
\text { mente tienen en cuenta las necesidades concretas del puesto de trabajo y se observa una } \\
\text { gran mejoría de los resultados. }\end{array}$ \\
\hline 9 & $\begin{array}{l}\text { No se obtiene evidencia. Los planes de formación que realiza este ayuntamiento nor- } \\
\text { malmente tienen en cuenta las necesidades concretas del puesto de trabajo y se observa } \\
\text { un gran empeoramiento de los resultados. }\end{array}$ \\
\hline
\end{tabular}

Fuente: elaboración propia. 
En relación con esta segunda proposición los encuestados comentan que aunque la preparación académica es necesaria para poder desempeñar adecuadamente un puesto de trabajo, también es necesario que los empleados de los ayuntamientos desarrollen unos valores y actitudes concretos para cada puesto. Según los entrevistados, estos comportamientos se pueden aprender con una adecuada formación.

La tercera proposición de investigación establece que las administraciones públicas que responsabilizan a los empleados públicos de la realización de su trabajo y evalúan su potencial obtienen mejores resultados que las que no lo hacen. En la tabla 5 se comenta cada caso de estudio, indicando si se obtiene evidencia o no sobre la proposición planteada.

Tabla 5

\section{Evidencia sobre la proposición de investigación 3}

\begin{tabular}{|c|c|}
\hline CASO & Evidencia \\
\hline 1 & $\begin{array}{l}\text { No se obtiene evidencia. Este ayuntamiento en ocasiones responsabiliza a los emplea- } \\
\text { dos de su trabajo y evalúa su potencial y se observa una gran mejoría de los resultados. }\end{array}$ \\
\hline 2 & No se puede afirmar la existencia de evidencia en uno u otro sentido. \\
\hline 3 & No se puede afirmar la existencia de evidencia en uno u otro sentido. \\
\hline 4 & $\begin{array}{l}\text { Se obtiene evidencia. Este ayuntamiento en ocasiones responsabiliza a los empleados } \\
\text { de su trabajo pero rara vez evalúa su potencial y se observa un empeoramiento de los } \\
\text { resultados. }\end{array}$ \\
\hline 5 & $\begin{array}{l}\text { Se obtiene evidencia. Este ayuntamiento en ocasiones responsabiliza a los empleados } \\
\text { de su trabajo pero casi nunca evalúa su potencial y se observa un empeoramiento de los } \\
\text { resultados a nivel de organización. }\end{array}$ \\
\hline 6 & $\begin{array}{l}\text { Se obtiene evidencia. Este ayuntamiento normalmente responsabiliza a los empleados } \\
\text { de su trabajo y evalúa su potencial y se observa una mejoría de los resultados a nivel de } \\
\text { prestación de servicios. }\end{array}$ \\
\hline 7 & $\begin{array}{l}\text { Se obtiene evidencia. Este ayuntamiento casi nunca responsabiliza a los empleados de } \\
\text { su trabajo ni evalúa su potencial y se observa un gran empeoramiento de los resultados } \\
\text { sobre todo a nivel de organización. }\end{array}$ \\
\hline 8 & $\begin{array}{l}\text { Se obtiene evidencia. Este ayuntamiento normalmente responsabiliza a los empleados } \\
\text { de su trabajo y evalúa su potencial y se observa una gran mejoría de los resultados. }\end{array}$ \\
\hline 9 & $\begin{array}{l}\text { Se obtiene evidencia. Este ayuntamiento casi nunca responsabiliza a los empleados de } \\
\text { su trabajo ni evalúa su potencial y se observa un gran empeoramiento de los resultados. }\end{array}$ \\
\hline
\end{tabular}

Fuente: elaboración propia.

Por lo que respecta a la tercera proposición de este trabajo, los encuestados en los ayuntamientos estudiados indican que es preciso que los objetivos a conseguir se expresen en términos de resultados concretos y no de metas genéricas. Señalan que, para vencer la reticencia natural que una evaluación del rendimiento genera, será necesario implantar la idea de que el establecimiento de los indicadores de gestión tiene como fin mejorar la gestión pública. Según la opinión de los encuestados, la flexibilización de estructuras, la 
descentralización del proceso decisorio, la implantación de una auténtica función directiva, la responsabilidad por los resultados obtenidos, la integración de la función de recursos humanos en un nivel estratégico, la valoración de las personas como un activo o la orientación hacia el servicio al ciudadano son algunas de las cuestiones que constituyen el punto de partida hacia un cambio de cultura organizativa en la Administración Pública que traiga consigo innovación y modernización de la gestión de los recursos humanos.

La cuarta proposición de investigación establece que las administraciones públicas que gestionan la retribución de sus empleados relacionándola con la evaluación del rendimiento obtienen mejores resultados que las que no lo hacen. En la tabla 6 se comenta cada caso de estudio, indicando si se obtiene evidencia o no sobre la proposición planteada.

Tabla 6

\section{Evidencia sobre la proposición de investigación 4}

\begin{tabular}{cl}
\hline CASO & \multicolumn{1}{c}{ Evidencia } \\
\hline 1 & $\begin{array}{l}\text { No se obtiene evidencia. Este ayuntamiento sólo en ocasiones relaciona la retribución } \\
\text { de sus empleados con la evaluación del rendimiento y, sin embargo, se observa una } \\
\text { gran mejoría de los resultados. }\end{array}$ \\
2 & No se puede afirmar la existencia de evidencia en uno u otro sentido. \\
3 & No se puede afirmar la existencia de evidencia en uno u otro sentido. \\
4 & Se obtiene evidencia. Este ayuntamiento sólo en ocasiones relaciona la retribución de \\
sus empleados con la evaluación del rendimiento y se observa un empeoramiento de los \\
resultados.
\end{tabular}

Fuente: elaboración propia.

Sobre la retribución, los estudios de casos analizados comentan que si la productividad no se refleja en el salario produce una gran desmotivación entre los empleados públicos y 
genera un rendimiento muy bajo. En su opinión, es necesario establecer un sistema en el que los trabajadores de los ayuntamientos vean que a mayor esfuerzo, mejores resultados y mayor recompensa.

A nivel general, los encuestados han puesto de manifiesto que las especiales características que el paradigma burocrático tiene para la gestión de recursos humanos en la Administración Pública la diferencian en gran medida de la gestión que se realiza en el ámbito privado. Este paradigma burocrático posee rasgos tales como la rígida jerarquía en la toma de decisiones, la existencia de múltiples niveles jerárquicos, la especialización minuciosa, el procedimentalismo, el carácter estatutario de la relación de empleo o la permanencia y garantía de por vida del puesto de trabajo en la Administración y, aunque actualmente se encuentra en crisis no existe, de momento, otro modelo que lo sustituya.

Como se puede observar, los resultados obtenidos al analizar las proposiciones de investigación que se han planteado el inicio de este trabajo no son determinantes pero aportan evidencia, con algunos matices, que apoyan las mismas. En la tabla 7 se resumen los resultados alcanzados en los estudios de casos.

Tabla 7

Resumen de la evidencia de los casos de estudio

\begin{tabular}{|c|c|c|}
\hline PROPOSICIÓN & $\begin{array}{c}\text { Sí } \\
\text { EVIDENCIA }\end{array}$ & $\begin{array}{c}\text { NO } \\
\text { EVIDENCIA }\end{array}$ \\
\hline $\begin{array}{l}\text { 1. Las administraciones públicas que en sus procesos de reclutamien- } \\
\text { to y selección tienen en cuenta las competencias concretas de los } \\
\text { puestos a cubrir obtienen mejores resultados que las que no lo hacen. }\end{array}$ & $\begin{array}{c}\text { Casos } 1,4 \\
6,7,8 \text { y } 9\end{array}$ & Caso 5 \\
\hline $\begin{array}{l}\text { 2. Las administraciones públicas que en sus planes de formación } \\
\text { tienen en cuenta las necesidades concretas del puesto de trabajo } \\
\text { obtienen mejores resultados que las que no lo hacen. }\end{array}$ & $\begin{array}{c}\text { Casos } 1,4 \\
6,7 \text { y } 8\end{array}$ & Caso 5 y 9 \\
\hline $\begin{array}{l}\text { 3. Las administraciones públicas que responsabilizan a los emplea- } \\
\text { dos públicos de la realización de su trabajo y evalúan su potencial } \\
\text { obtienen mejores resultados que las que no lo hacen. }\end{array}$ & $\begin{array}{c}\text { Casos } 4,5 \\
6,7,8 \text { y } 9\end{array}$ & Caso 1 \\
\hline $\begin{array}{l}\text { 4. Las administraciones públicas que gestionan la retribución de } \\
\text { sus empleados relacionándola con la evaluación del rendimiento } \\
\text { obtienen mejores resultados que las que no lo hacen. }\end{array}$ & $\begin{array}{c}\text { Casos } 4,6, \\
7 \text { y } 9\end{array}$ & $\begin{array}{c}\text { Caso } 1,5 \\
\text { y } 8\end{array}$ \\
\hline
\end{tabular}

Fuente: elaboración propia.

\section{CONCLUSIONES}

En la actualidad existe una importante corriente en la literatura preocupada por demostrar el impacto de la gestión de recursos humanos sobre los resultados organizativos (Russell et al., 1985; Huselid, 1995; Delaney y Huselid, 1996; Delery y Doty, 1996; Huselid et al., 1997; Ichniowski y Shaw, 1999; Zheng et al., 2006; Ghebregiorgis y Karsten, 2007). Asimismo, las empresas muestran un creciente interés por sus trabajadores y por las prácticas relacionadas con su gestión. Este interés puede estar explicado por la aceptación 
generalizada de que los recursos humanos son una de las principales fuentes de ventaja competitiva sostenible.

Para tratar de demostrar este efecto positivo de una adecuada gestión de recursos humanos se han realizado numerosos trabajos, la mayoría de los cuales se han centrado en el ámbito privado, dejando en un segundo plano el ámbito de la Administración Pública. Para cubrir este hueco y entender mejor esta relación, en este trabajo se ha examinado el impacto que diversas prácticas de recursos humanos tienen sobre los resultados de las corporaciones locales.

El análisis de nueve ayuntamientos de la Región de Murcia apoya la idea de que efectivamente una adecuada gestión de las prácticas de recursos humanos tiene efecto en los resultados de los ayuntamientos. De hecho, aunque hay prácticas que están muy reguladas por la normativa de la función pública se pueden gestionar de forma más eficiente sin colisionar con la normativa vigente. En este estudio se encuentra evidencia de que aquellos ayuntamientos que muestran mayor preocupación por una dirección adecuada de sus recursos humanos, estableciendo procesos de reclutamiento y selección que tienen en cuenta las competencias concretas de los puestos a cubrir, realizando planes de formación basados en las necesidades específicas del puesto de trabajo, responsabilizando a los empleados públicos de su trabajo, evaluando el potencial de sus trabajadores y relacionando la retribución con la evaluación del rendimiento, están obteniendo una mejor evolución de sus resultados en los tres niveles analizados: de organización, de trabajador y de prestación de servicios. Mientras, aquellas corporaciones locales que presentan menor interés por la gestión de sus trabajadores muestran una trayectoria más negativa de sus indicadores.

Como se ha puesto de manifiesto en el apartado de los resultados, durante la realización de los estudios de casos se ha recogido la opinión de los participantes, ya que se considera que pueden aportar buenas ideas para mejorar la gestión de los recursos humanos en los ayuntamientos. En general, los entrevistados señalan que las grandes líneas de actuación en los procesos de modernización y mejora de la calidad en los servicios públicos pasan por reforzar actuaciones en materia de recursos humanos, lo que debe ser tenido en cuenta por los poderes públicos.

Con este trabajo se espera haber contribuido a que se valore el importante papel que los recursos humanos desempeñan en el buen funcionamiento de las corporaciones locales y a mostrar posibles líneas de actuación.

La principal limitación de este estudio es la que se deriva de la metodología empleada. El estudio de casos resulta adecuado para abordar temas escasamente investigados y profundizar en las variables que pueden afectarles pero sus resultados no son generalizables. No obstante, creemos que sus conclusiones son interesantes ya que recogen opiniones de personas que conocen en profundidad la problemática tratada. Otra limitación es la de que los resultados obtenidos podrían estar condicionados por las características de los ayuntamientos que forman la muestra y puede existir un sesgo en la respuesta al haber contestado sobre sus propios resultados.

Por tanto, las futuras líneas de investigación que se proponen van dirigidas, fundamentalmente, a subsanar estas limitaciones del estudio. Así, para superar las limitaciones de un estudio cualitativo se recomienda un análisis cuantitativo, ampliando la población objeto del trabajo a todos los ayuntamientos del territorio nacional. Asimismo, se propone recoger datos no sólo desde el punto de vista de los ayuntamientos sino de otros colectivos relacionados con el tema, como puede ser el de la ciudadanía. 


\section{REFERENCIAS BIBLIOGRÁFICAS}

ALAVI, M. Y LEIDNER, D.E. (2001): "Review: knowledge management and knowledge management systems: conceptual foundations and research issues”, MIS Quarterly, Vol. 25, No. 1, pp. 107-136.

AMIT, R. Y SCHOEMACKER, P.J.H. (1993): "Strategic assets and organizational rent", Strategic Management Journal, Vol. 14, No., pp. 33-46.

AMMONS, D. (1995): "Overcoming the inadequacies of performance measurement in local government: the case of libraries and leisure services", Public Administration Review, Vol. 55, No. 1, pp. 4-8.

ANDREU, I. (2009): “Modernización y nueva gestión pública en los ayuntamientos del Camp de Tarragona", Tesis doctoral.

ARAGÓN, A.; BARBA, M.I. Y SANZ, R. (2003): “Effects of Training on Business Results”, The International Journal of Human Resource Management Vol. 14, pp. 956-980.

ARTHUR, J.B. (1994): "Effects of human resource systems on manufacturing performance and turnover", Academy of Management Journal, Vol. 37, No. 3, pp. 670-687.

BAE, J. Y LAWLER, J.J. (2000): “Organizational and HRM strategies in Korea: Impact on firm performance in an emerging economy", Academy of Management Journal, Vol. 43, No. 3, pp. 502-519.

BARNEY J.B. Y WRIGHT P.M. (1998): “On becoming a strategic partner: the role of human resources in gaining competitive advantage “, Human Resource Management, Vol. 37, No. 1, pp. 31-46.

BARNEY, J.B. (1991): "Firm resources and sustained competitive advantage ", Journal of Management, Vol. 17, pp. 99-120.

BARRETT, A. Y O'CONNELL, P.J. (2001): "Does training generally work? The returns to incompany training”, Industrial \& Labor Relations Review, Vol. 54, No. 3, pp. 647.

BARRON, J.M. Y BERGER, M.C. (1999): “Do workers pay for on-the-job training?”, Journal of Human Resources, Vol. 34, No. 2, pp. 235-253.

BARTEL, A.P. (1994): "Productivity Gains from the Implementation of Employee Training Programs", Industrial Relations, Vol. 33, No. 4, pp. 411-425.

BATT, R. (2002): "Managing customer services: Human resource practices, quit rates, and sales growth", Academy of Management Journal, Vol. 39, pp. 779-801.

BIRDI, K.; CLEGG, C.; PATTERSON M.; ROBINSON A.; STRIDE C,.B.; WALL, T.D. Y WOOD, S.J. (2008): "The impact of human resource and operational management practices on company productivity: A longitudinal study", Personnel Psychology, Vol. 61, pp. 467-501.

BLACK, S. Y LYNCH, L. (1996): "Human capital investments and productivity", American Economic Review, Vol. 86, No. 2, pp. 263-267.

BOLLINGER, A.S. Y SMITH, R.D. (2001): "Managing organizational knowledge as a strategic asset”, Journal of Knowledge Management, Vol. 5, No. 1, pp. 8-18.

BOSELIE, P.; PAAUWE, J. Y JANSEN, P. (2001): "Human resource management and performance: Lessons from The Netherlands", International Journal of Human Resource Management, Vol. 12, No. 7, pp. 1107-1125.

BOWMAN, J.S. (1994): "At last, an alternative to performance appraisal: total quality management”, Public Administration Review, Vol. 54, No. 2, pp. 129-136.

CARUTH, D.L. Y HUMPHREYS, J.H. (2008): "Perfomance appraisal: Essential characteristics for strategic control", Measuring Business Excellence, Vol. 12, No. 3, pp. 24-32.

CHIU, R.K.; LUK, V.W.N. Y TANG, T.L. (2002): "Retaining and motivating employees: Compensation preferences in Hong Kong and China", Personnel Review, Vol. 31, pp. 402-431.

COGGBURN, J.D. Y HAYS, S.W. (2003): "Innovations in Local Government human resource systems: observations from several best practice locations", Public Administration Quarterly, Vol. 23, No. $3 / 4$, pp. $433-455$. 
DARKE, P.; SHANKS, G. Y BROADBENT, M. (1998): "Successfully completing case study research: combining rigour, relevance and pragmatism", Information Systems Journal, Vol. 8, pp. 273-289.

DE LA CALLE, M.C. Y ORTIZ DE URBINA, M. (2004): Fundamentos de recursos humanos, Madrid, Ed. Prentice Hall.

DE LANCER JULNES, P. Y HOLZER, M. (2003): "Promoting the utilization of performance measures in public organisations: an empirical study of factor affecting adoption and implementation", Public Administration Review, Vol. 61, No. 5, pp. 693-708.

DELANEY, J. Y HUSELID, M. (1996): “The Impact of Human Resources Management Practices on Perceptions of Organizational Performance", Academy of Management Journal, Vol. 39, No. 4, pp. 949-969.

DELERY, J.E. Y DOTY, D.H. (1996): "Modes of theorizing in strategic human resource management: tests of universalistic, contingency and configurational performance predictions ", Academy of Management Journal, Vol. 39, No. 4, pp. 802-835.

DOLAN, S.L.; VALLE, R.; JACKSON, S.E. Y SCHULER, R.S. (2003): La gestión de recursos humanos, Madrid, Ed. McGraw-Hill.

FAEMS, D.; SELS, L.; DEWINNE, S. Y MAES, J. (2005): "The effect of individual HR domains on financial performance", The International Journal of Human Resource Management, Vol. 16, pp. 676-700.

GABRIS, G.T.; GRENELL, K.D. Y KAATZ, J. (1998): "Reinventing Local Government human services management: A conceptual analysis", Public Administration Quarterly, Vol. 22, No. 1, pp. 74-96.

GARCÍA, M.I. (2007): "La nueva gestión pública: evolución y tendencias”, Presupuesto y Gasto Público, No. 47, pp. 37-64.

GHEBREGIORGIS, F. Y KARSTEN, L. (2007): "Human resource management and performance in a developing country", The International Journal of Human Resource Management, Vol. 18, pp. 321-332.

GÓMEZ-MEJÍA, L.R.; BALKIN, D.B. Y CARDY, R.L. (2001): Dirección y gestión de recursos humanos, Madrid, Ed. Prentice-Hall.

GONZALO, J. Y CORTÉS, J.V. (2004): "Modernización y cambio en la gestión de los recursos humanos en la administración local", Capital Humano, Vol. 176, pp. 58-66.

GRAY, G. (2002): “Performance appraisals don't work”, Industrial Management, Vol. 44, No. 2, pp. 15-17.

HENDERSON, R.L. (2003): Compensation management in a Knowledge-based world, New Jersey, Ed. Prentice-Hall.

HITT, M.A.; BIERMAN, L.; SHIMIZU, K. Y KOCHHA, R.R. (2001): "Direct and moderating effects of human capital on strategy and performance in professional service-firms: a resourcebased perspective", Academy of Management Journal, Vol. 44, No. 2, pp. 13-28.

HOLZER H.; BLOCK R.; CHEATHAM, M. Y KNOTT, J. (1993): "Are training subsides for firms effective? The Michigan experience", Industrial \& Labor Relations Review, Vol. 46, No. 4, pp. 625-636.

HUSELID, M.A. (1995): "The impact of human resource management practices on turnover, productivity and corporate financial performance “, Academy of Management Journal, Vol. 38, No. 3, pp. 635-672.

HUSELID, M.A.; JACKSON, S.E. Y SCHULER, R.S. (1997): “Technical and strategic human resource management effectiveness as determinants of firm performance", Academy of Management Journal, Vol. 40, No. 1, pp. 171-188.

ICHNIOWSKI, C. Y SHAW, K. (1999): “The effects of human resource management systems on economic performance: An international comparison of U.S. and Japanese plants", Management Science, Vol. 45, No. 5, pp. 704-721. 
ICHNIOWSKI, C.; SHAW, K. Y PRENNUSHI, G. (1997): "The effects of human resource management practices on productivity: a study of steel finishing lines", The American Economic Review, Vol. 87, No. 3, pp. 291-313.

JERÉZ GÓMEZ, P., CESPEDES LORENTE, J.J. Y VALLE CABRERA, R. (2004): “Training practices and organisational learning capability", Journal of European Industrial Training, Vol. 28, No. 2-4, pp. 234-256.

KAMOCHE, K. (1996): "Strategic human resource management within a resource-capability view of the firm", Journal of Management Studies, Vol. 33, No. 2, pp. 213-233.

KHOURY, G.C. Y ANALOUI, F. (2004): "Innovative management model for performance appraisal: The case of the Palestinian public universities", Management Research News, Vol. 27, No. 1, pp. 56-73.

KIDDER, P. J. Y ROUILLER, J.Z. (1997): "Evaluating the Success of a Large-Scale Training Effort", National Productivity Review, Vol. Spring, pp. 79-89.

KIM, S. (2005): "Factors affecting state government information technology employee turnover intentions", American Review of Public Administration, Vol. 35, pp. 137-156.

KOCH, M.J. Y MCGRATH, R.G. (1996): "Improving labour productivity: Human resource management policies do matter", Strategic Management Journal, Vol. 17, pp. 335-354.

KRAUCHUCK, R.S. Y SCHACK, R. (1996): "Designing effective performance measurement systems under the government performance and results act of 1993", Public Administration Review, Vol. 56, No. 4, pp. 348-358.

LARRAZA KINTANA, M.; URTASUN ALONSO, A. Y GARCÍA OLAVERRI, C. (2006): "Highperformance work systems and firms' operational performance: The moderating role of technology", The International Journal of Human Resourc Management, Vol. 17, No. 1, pp. 70-85.

LATORRE, J.A. (2003): "La formación como mejora del empleo público: la experiencia de la Diputación de Alicante”, Análisis Local, Vol. 46, pp. 51-54.

LAWLER, E.E. (1990): Strategic pay. Aligning organizational strategies and pay systems, San Francisco, Ed. Jossey-Bass.

LENGERMANN, P.A. (1996): "The benefits and costs of training: a comparison of formal company training, vendor training, outside seminars, and school based training “, Human Resource Management, Vol. 35, pp. 361-381.

LEWIS, G.B. (1991): "Turnover and the quiet crisis in the federal service", Public Administration Review, Vol. 51, pp. 145-155.

LUNA, A. (2004): "Gestión del capital humano e intelectual en las Adminsitraciones Públicas: El primer paso para la eficiencia del sector público", Capital Humano, Vol. 176, pp. 105-106.

MANI, B.G. (2002): "Performance appraisal systems, productivity, and motivation: A case study", Public Personnel Management, Vol. 31, No. 2, pp. 141-159.

MARTIN, D.C. Y BARTOL, K.M. (1998): "Performance appraisal: Maintaining systems effectiveness", Public Personnel Management, Vol. 27, No. 2, pp. 223-231.

MARTOCCHIO, J.J. (1998): Strategic compensation: A human resource management approach, New Jersey, Ed. Prentice Hall.

MASTRACCI, S.H. (2009): "Evaluating HR management strategies for recruiting and retaining IT professionals in the U.S. federal government", Public Personnel Management, Vol. 38, No. 2, pp. 19-34.

MCELROY, J.C. (2001): "Managing workplace commitment by putting people first", Human Resource Management Review, Vol. 11, No. 3, pp. 327-335.

MONDY, R.W.; NOE, R.M. Y PREMEAUX S.R. (1999): Human resource management, New Jersey, Ed. Prentice-Hall.

MUELLER, F. (1996): "Human resources as strategic assets: an evolutionary resource-based theory", Journal of Management Studies, Vol. 33, No. 6, pp. 757-785. 
MURRAY, B. Y RAFFAELE, G.C. (1997): "Single-site, results-level. Evaluation of Quality awareness training", Human Resource Development Quarterly, Vol. 8, No. 3, pp. 229-245.

PARRÉS, A. (2005): "El reto de la "Nueva Gestión Pública" a través de un cambio basado en las personas", Auditoría Pública, No. 37, pp. 57-63.

PFEFFER, J. (1995): "Producing sustainable competitive advantage through the effective management of people", Academy of Management Executive, Vol. 9, No. 1, pp. 55-72.

RAGHURAM, S. (1994): "Linking staffing and training practices with business strategy: A theoretical perspective", Human Resource Development Quarterly, Vol. 5, No. 3, pp. 237-251.

RUSAW, C. (2009): "Professionalism under the "Performance-based pay" reform: A critical assessment and alternative development model", Public Personnel Management, Vol. 8, No. 4, pp. 35-54.

RUSSELL, J.S.; TERBORG, J.R. Y POWERS, M.L. (1985): “Organizational performance and organizational level training and support", Personnel Psychology, Vol. 38, pp. 849-863.

SÁENZ BLANCO, M.T. (2004): "El sistema de gestión por competencias del Ayuntamiento de Manlleu: Una alternativa de evaluación para el desempeño excelente", Capital Humano, Vol. 176, pp. 98-104.

SANGER, M.B. (2008): "From measurement to management: breaking through the barriers to state and local performance", Public Administration Review, Vol. 68, No. 1, pp. 70-85.

SANZ VALLE, R. Y SABATER SÁNCHEZ, R. (2002): "Enfoque universalista de recursos humanos. Estudio empírico de sus efectos sobre los resultados de la empresa”, Revista Europea de Dirección y Economía de la Empresa, Vol. 11, No. 1, pp. 163-182.

SCHONEWILLE, M. (2001): "Does training generally work? Explaining labour productivity effects from schooling and training”, International Journal of Manpower, Vol. 22, No. 1/2, pp. 158-172.

SOLE, F. Y SCHIUMA, G. (2010): "Using performance measures in public organisations challenges of Italian public administration”, Measuring Business Excellence, Vol. 14, No. 3, pp. 70-84.

TERPSTRA, D.E. Y ROZELL, E.J. (1993): "The relatioship of staffing practices to organisational level measures of performance", Personnel Psychology, Vol. 46, pp. 27-48.

UBEDA GARCÍA, M. (2005): "Training and business performance: The Spanish case", International Journal of Human Resource Management, Vol. 16, pp. 1691-1710.

UDECHUKWU, I. Y MANYAK, T. (2009): "Job applicants' perceptions of resumes versus employment application forms in the recruitment process in a pubis organization", Public Personnel Management, Vol. 38, No. 4, pp. 79-96.

UFUK, K. (2007): "Performance management for public personnel: A multy-analysis approach toward personnel”, Public Personnel Management, Vol. 36, No. 2, pp. 93-113.

VILLORIA MENDIETA, M.; Y DEL PINO MATUTE, E. (2009). Dirección y gestión de recursos humanos en las administraciones públicas, Madrid, Ed. Tecnos.

WRIGHT, P.M.; MCMAHAN, G.C. Y MCWILLIAMS, A. (1994): "Human resources and sustained competitive advantage: a resource-based perspective", International Journal of Human Resource Management, Vol. 5, No. 2, pp. 301-326.

YIN, R.K. (1994): Case study research: Design and methods, London, Ed. Sage Publications.

YOUNDT, M.A.; SNELL, S.A.; DEAN, J.W. Y LEPAK, D.P. (1996): "Human resource management, manufacturing strategy, and firm performance", Academy of Management Journal, Vol. 39, No. 4, pp. 836-866.

ZHENG, C.; MORRISON, M. Y GRANT O. (2006): "An empirical study of high performance HRM practices in Chinese SMEs", International Journal of Human Resource Management, Vol. 17, No. 10, pp. 1772-1803. 